\title{
Error in visually directed manual pointing
}

\author{
J. M. FOLEY \\ University of California, Santa Barbara, California 93106
}

\begin{abstract}
Errors in pointing at visual targets without sight of the hand or arm were measured for both hands in two cue conditions. The existence of large errors of overreaching, which increase as cues are reduced, was confirmed. These vary with target distance but not appreciably with the hand used or the target direction. Large lateral errors were also found. These can be reasonably well described by rotations about the midpoint of the eyes. Their magnitude and direction depend on the subject and the hand used, but are largely independent of cue condition and distance. The mean angular error across subjects taken without regard to sign was $4.8 \mathrm{deg}$. The mean difference between hands was $5.2 \mathrm{deg}$. The effect of eye dominance expected under the hypothesis of Walls and Ogle was not obtained.
\end{abstract}

Visually directed pointing, as contrasted to visually guided pointing, refers to pointing at a visual target without the opportunity to view the hand or arm. Foley and Held (1972) found that large errors occur in this task. They found a general tendency to overreach targets. Overreaching was very great, about $25 \mathrm{~cm}$, when convergence was the only cue to distance. However, consistent overreaching was also found when there were many cues to distance. Large angular errors of up to $10 \mathrm{deg}$ were also found. When the favored hand was used, these errors were in almost all cases to the side opposite the sighting eye. This result was consistent with the hypothesis of Walls (1951) and Ogle (1962) that the perceived direction of a target is its direction from the dominant eye. However, of the seven subjects who pointed with their favored hand, five had their favored hand on the same side as their sighting eye. Thus, evidence for an effect of the sighting eye comes from two subjects only. Furthermore, in two cases where pointing was done with the nonfavored hand, the error was on the side opposite the hand used and not opposite the sighting eye. The study thus suggested that there are two factors which influence angular error, sighting eye, and hand used.

The present study was designed to clarify further the facts about pointing error. There are two principal differences from the earlier study. (1) Each subject used both his left and his right hand. (2) Stimuli to convergence and accommodation varying together replaced the convergence condition of the earlier study in which the stimulus to convergence varied while the stimulus to accommodation was fixed at $75 \mathrm{~cm}$. Unlike in the convergence condition of Foley and Held, any overreaching found in the accommodation-convergence condition cannot be attributed to a lack of agreement between the two cues.

This research was supported by USPHS Grant EY-00666 from the National Eye Institute. The author is grateful to Theodore H. Applebaum for his assistance in this research.

\section{METHOD}

The subject was seated directly in front of a horizontal board that completely occluded his view of his hand and arm. His head was held in place by means of a bite made of dental impression compound. The bottom surface of the board was about $10 \mathrm{~cm}$ below eye level. Over the index finger of the hand being used, the subject wore a rubber cap from which a metal probe extended $.5 \mathrm{~cm}$. His task was to look at the visual target and to touch the probe to the bottom of the board directly below the perceived position of the target. The two coordinates of the pointed position were read out electronically by means of a device similar to that described by Bauer, Woods, and Held (1969).

There were two conditions with respect to the spatial information available in the stimulus, an accommodation-convergence condition and a multicue condition. In the accommodationconvergence condition, two lights, each visible to only one eye, were used to simulate a single point-source, viewed binocularly. The lights moved along a horizontal line at eye level in a frontal plane $50 \mathrm{~cm}$ from the nodal points of the eyes (taken to be $0.7 \mathrm{~cm}$ behind the corneas). Suitably oriented polarizers in front of each eye and in front of each light allowed the right eye to be stimulated only by the left light, and vice versa. The effect simulated a single point of light situated at the intersection of the lines joining each light with the nodal point of the eye to which it was visible. The distance of this simulated point was varied by moving the lights together or apart, and its direction, by moving both lights left or right. The stimulus to accommodation was made to agree with the stimulus to convergence by viewing the stimulus through negative lenses appropriate to the simulated distance. This method of presentation allows the stimuli to accommodation and convergence to vary together while holding retinal size and illumination essentially constant and eliminating other distance cues.

The stimuli were Bausch and Lomb ophthalmoscope bulbs having coil filaments less than $1 \mathrm{~mm}$ in diam. Their intensity was approximately $10^{-4} \mathrm{~cd}$ (about $2 \mathrm{log}$ units above foveal threshold). They had the appearance of bright stars seen against a background of total darkness. There were 12 target positions, 6 each along lines extending in distance $12 \mathrm{deg}$ to the left and right of the median plane. The range of target distances was approximately $15-40 \mathrm{~cm}$, with slight variations from subject to subject, depending on interpupillary distance.

In the multicue condition, the stimuli were plastic rods, $0.5 \mathrm{~cm}$ in diam and $1.25 \mathrm{~cm}$ in height, with a slightly larger $\mathrm{knob}$ at the top and bottom (pushpins). These were viewed in an illuminated field. They stood on a cloth patterned in black, white, and gray squares about $0.5 \mathrm{~cm}$ on a side. This surface provided perspective cues. Stimuli to accommodation and convergence and binocular disparity were also available. A single target rod was present on each trial. It was presented in a set of positions that corresponded approximately 
to the set of simulated positions in the accommodation-convergence condition.

Eight subjects participated in this experiment. All were young adults with acuity of $20 / 20$ and stereoacuity of $6 \mathrm{sec}$ of arc or better, as measured by Keystone Orthorater tests. Four subjects were right-handed and four were left-handed. For five subjects, sighting eye was on the same side as the favored hand; for three subjects, it was on the opposite side. Sighting eye was determined in the following way. The subject pointed at a target 2 or $3 \mathrm{~m}$ away, then closed one eye at a time and noted which eye was lined up with the pcinting finger and the target. In every case, the same cye was reported to be so aligned regardless of the hand used for pointing.

The experimental design was as follows. All subjects responded to both the accommodation-convergence and the multicue conditions with both left and right hands. There were eight conditions in all ( 2 cue conditions by 2 directions by 2 hands) with six target distances in each. At each session, three consecutive responses were made to each target in each condition. The order of the conditions was counterbalanced and, within a condition, the order of the target distances was randomized. One practice session preceded the experiment, except for those subjects who were already experienced at this kind of task. The experiment consisted of six sessions, yielding 18 responses to each target.

\section{RESULTS}

Mean pointed position was determined for each target distance in each condition for each subject. These mean pointed positions were used to compute a distance error (pointed distance minus target distance, with distance measured from the frontal plane through the nodal points to the frontal plane through the pointed position and target, respectively) and an angular error (pointed direction minus target direction, where both directions were measured from the midpoint between the nodal points). Each error measure was analyzed separately in a four-way analysis of variance (Distance by Cue Condition by Direction by Hand) to determine which variables had significant effects across subjects.

\section{Distance Error}

Figure 1 shows mean pointed distance as a function of target distance taken over the eight subjects and two target directions with cue condition and hand (favored vs. unfavored) as parameters. Errors of overreaching were found in agreement with Foley and Held (1972).

Cue condition. Distance error was very significantly greater in the accommodation-convergence condition than in the multicue condition $(F=60.8 ; \mathrm{df}=1,7$; $\mathrm{p}<.001$ ).

Target distance. Distance error decreased significantly as distance increased $(\mathrm{F}=13.2$, df $=$ $3,28 ; \mathrm{p}<.001)$. This decrease is almost entirely in the accommodation-convergence condition, resulting in a significant Cue Condition by Distance interaction ( $F$ $=11.9 ; \mathrm{df}=4,28 ; \mathrm{p}<.001)$.

Target direction. There was a small, but significant, tendency for subjects to overreach targets in the left field more $(F=16.7 ; \mathrm{df}=1,7 ; \mathrm{p}=.005)$.

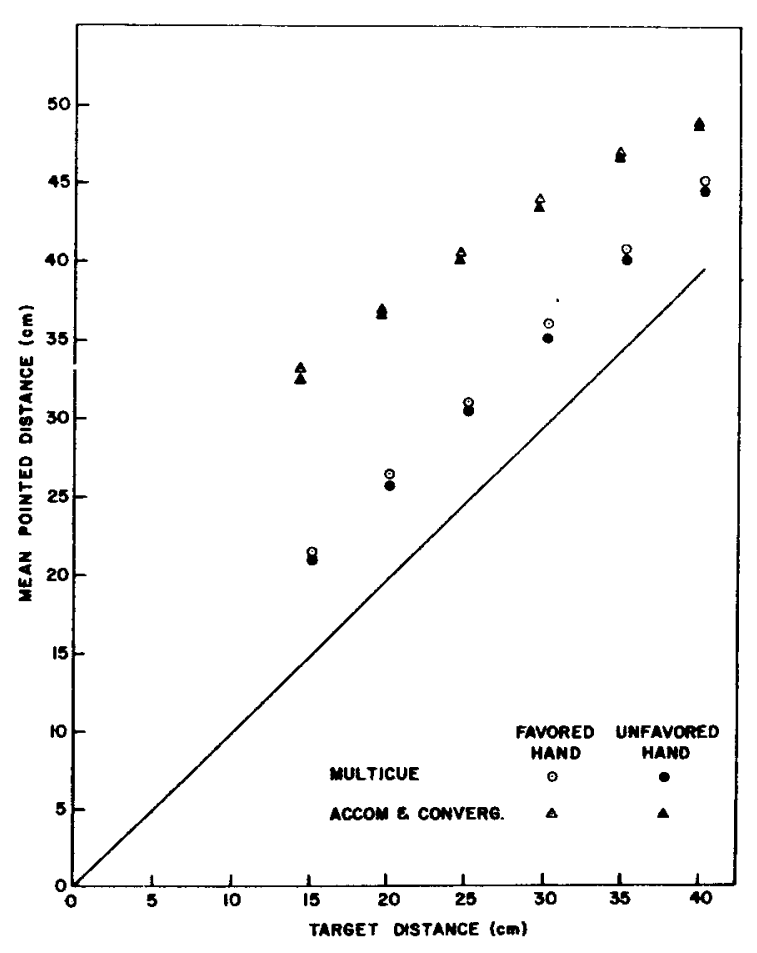

Figure 1. Grand mean pointed distance as a function of target distance taken over the eight subjects with cue condition and hand (favored vs. unfavored) as parameters. Diagonal line indicates target distance.

The mean difference in error was $1.6 \mathrm{~cm}$. This effect is at least partially attributable to the fact that there was an overall rightward angular error (see below), which means that, measured in terms of radial distance, this difference would be smaller.

Hand. No significant difference was found between left and right hands $(\mathrm{F}=.7 ; \mathrm{df}=1,7 ; \mathrm{p}=.43)$. However, as is shown in Figure 1, the favored hand reached about $.5 \mathrm{~cm}$ farther than the unfavored hand on the average.

Individual differences. Although the analysis did not test for individual differences, these are obviously significant. All subjects, however, consistently overreached in the accommodation-convergence condition. In the multicue condition, the error was always less. For a few subjects it was near zero, but no subject showed a consistent tendency to underreach. No subject had a mean difference between his two hands larger than $2.9 \mathrm{~cm}$.

\section{Angular Error}

Figure 2 shows the mean pointed position for all subjects, the mean being taken over subjects with target direction and hand as parameters. The multicue condition is represented in the left panel and the accommodation-convergence condition in the right. 


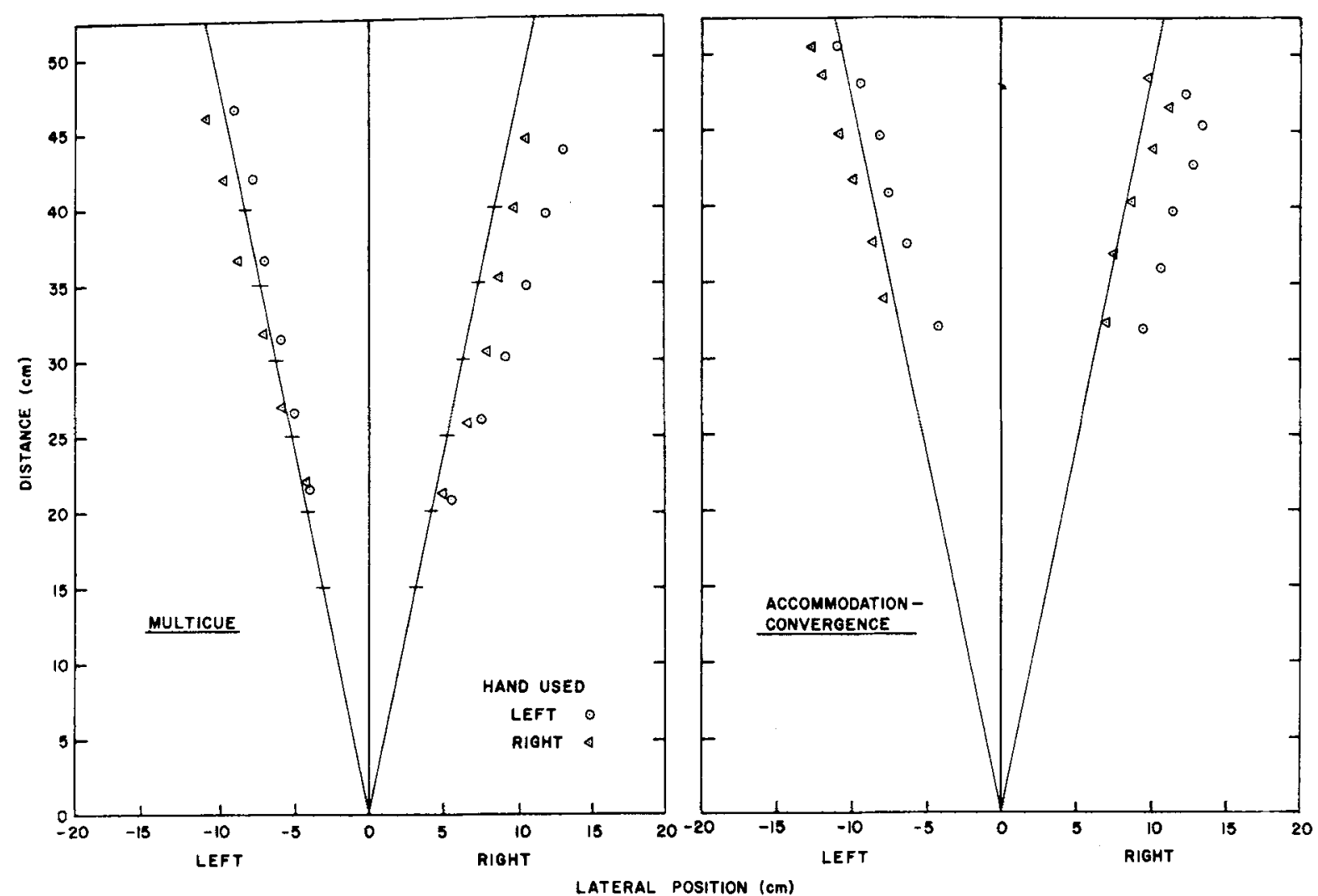

Figure 2. Grand mean pointed position as a function of hand, target direction, and cue condition taken across all subjects. Target directions are indicated by the radial lines going out from the origin to the left and right of the miedian plane; target positions, by horizontal slashes along these lines; left-hand responses by circles, and right-hand responses by triangles.

Cue condition. The effect of cue condition on angular error was not significant $(F=1.4$; $\mathrm{df}=1,7$; $\mathrm{p}=.28$ ). Since this same variable has a large effect on pointed distance, it follows that the angular error is independent of the distance error for a particular target position.

Target distance. Target distance had a small, but significant, effect on angular error $(F=4.0$; $\mathrm{df}=$ $4,28 ; p<.05)$. This means that there is a portion of the angular error which cannot be accounted for by a constant rotation about the mid point of the eyes. This distance effect can be described as a tendency for targets to be localized directionally farther from ${ }^{-}$the median plane as distance increases. In Figure 2, this is most evident for the right hand and in the left field, but the effect is small.

Target direction. Angular error is significantly larger in the right field $(F=29.3$; df $=1,7$; $\mathrm{p}<.001)$. This error is to the right, and its mean value is $3.4 \mathrm{deg}$.

Hand. Although a consistent tendency for the right hand to point to the left of the left hand is evident in Figure 2, since there are large individual differences, this effect is not statistically significant across subjects. Two of the eight subjects show large effects in the opposite direction. This lack of consistency across subjects does not mean that hand effects for individual subjects are small or insignificant. Mean angular errors taken across cue condition and target direction are presented for individual subjects in Table 1. The mean of the absolute values of the differences between the two hands is 5.2.

Individual differences. It is evident that individual differences are highly significant. Mean overall error is given for each subject in Table 1 . The mean of the absolute values of these errors is $4.8 \mathrm{deg}$. The errors for right-eye-dominant subjects and left-eye-dominant subjects are grouped separately for purposes of comparison. It is clear that the mean angular error for the left-eye-dominant subjects is more to the left than that of the right-eye-dominant subjects, contrary to the expectation derived from the Walls-Ogle hypothesis. The largest effect evident in the table is the tendency for the angular error to be toward the side of the dominant hand. The right-handed subjects reached an average of $6.9 \mathrm{deg}$ to the right of the left-handed subjects. This effect is not statistically significant, however $(\mathrm{t}=2.01 ; \mathrm{df}=3 ; \mathrm{p}>.05)$. 
There is no tendency for the favored hand to produce less angular error than the unfavored hand.

\section{DISCUSSION}

The results reveal a clear separation between distance error and angular error with respect to the variables that affect them. Most salient are the facts that (1) cue condition has a large effect on distance error and no significant effect on angular error, and (2) the hand used has a very small effect on the distance error and a large effect on angular error for individual subjects.

With respect to distance error, these results are consistent with those of Foley and Held (1972). The present study has shown further that large errors of overreaching do not depend on the lack of agreement between accommodation and convergence that existed in the Foley-Held study. These results also establish an interaction between cue condition and distance, with the distance error in the accommodationconvergence condition tending to decrease as distance increases, while error in the multicue condition remains approximately constant. These results are consistent with the suggestions made by Foley and Held (1972) that (1) the differences in distance error between the cue conditions are mediated by different distance signals produced in the visual system, and (2) these different distant signals may result from the interaction of the cues with the specific distance tendency (Gogel, 1972).

Angular error is less easily accounted for. Foley and Held (1972) obtained some evidence for an effect of dominant eye consistent with the Walls-Ogle hypothesis. Walls (1951) and Ogle (1962) hypothesized that visual direction is the direction from the dominant eye as determined by sighting tests similar to the one used to establish eye dominance in the present study. If this is the case, when there is overreaching the subject will err to the side opposite the dominant eye. In general, this effect will be small and will be largest when the target is near and the overreaching error is large. In the present study, the mean angular error for the two groups of subjects is in the direction opposite to that predicted by the hypothesis. Also, the predicted relation with overreaching error was not found. Therefore, the results do not provide any support for the Wall-Ogle hypothesis. Nor do they provide sufficient evidence to reject it, since, due to the large variance resulting from individual differences, the test was not sufficiently sensitive to detect the small effect predicted by this hypothesis.

There is other evidence, however, which casts doubt on the Walls-Ogle hypothesis. The experiment on which this hypothesis is based is attributed by Helmholtz to Hering and was later repeated by Walls
Table 1

Mean Angular Error for Individual Subjects in Degrees

\begin{tabular}{lrrrrr}
\hline $\begin{array}{c}\text { Right-Eye } \\
\begin{array}{c}\text { Dominant } \\
\text { Subjects }\end{array}\end{array}$ & $\begin{array}{c}\text { Favored } \\
\text { Hand }\end{array}$ & $\begin{array}{c}\text { Right- } \\
\text { Hand } \\
\text { Error }\end{array}$ & $\begin{array}{c}\text { Left- } \\
\text { Hand } \\
\text { Error }\end{array}$ & $\begin{array}{c}\text { Diffe- } \\
\text { rence } \\
\text { L - R }\end{array}$ & Mean \\
\hline F. S. & R & -1.3 & 5.6 & 6.9 & 2.2 \\
L. F. & R & 7.7 & 3.3 & -4.4 & 5.5 \\
S. E. & R & 11.2 & 8.3 & -2.9 & 9.8 \\
C. M. & L & -2.2 & -3.9 & -1.7 & -3.1 \\
T. C. & L & .4 & -1.3 & -1.7 & -.5 \\
Left-Eye & & & & & \\
Dominant & & & & & \\
Subjects & & & & & \\
C. K. & L & -15.7 & -1.6 & 14.1 & -8.7 \\
M. P. & L & 3.5 & 7.0 & 3.5 & 5.3 \\
D. H. & R & .2 & 6.0 & 6.2 & 2.9 \\
Mean X & & & & 2.5 & 1.7 \\
Mean IX & & & & 5.2 & 4.8 \\
\hline
\end{tabular}

Note-Error was averaged over distance, direction, and cue condition. Rightward errors are designated by positive numbers, leftward by negative numbers.

(1951) and Ogle (1962). In it, a subject binocularly fixates a near point of light. This is extinguished and replaced by a farther target which is seen by one eye only, the other eye being covered. This second target is in alignment with the first target and with the viewing eye. It is assumed that the changed stimulus to accommodation causes the unstimulated eye to turn so that both eyes are converged at the new target distance (accommodative vergence). Both Walls and Ogle found that a large portion of their subjects reported a lateral shift in the position of the second target only when one eye was covered, but not the other. They concluded that subjective visual direction was determined by direction with respect to one eye only. The author has tried unsuccessfully to determine the sighting dominant eye using this procedure. Of seven subjects tested, all of whom had shown clear dominance in the simple sighting test described above (see Method section), every one reported lateral movement in the expected direction regardless of which eye was covered. These subjects also pointed at the binocular target and the two monocular targets. In all but one case, the binocular target was located intermediate in direction with respect to the two monocular lights, although it did tend to be closer in direction to the monocular target seen by the dominant eye. This suggests that the effect of the sighting dominant eye on angular error may be very much smaller than the Walls-Ogle hypothesis would predict. A recent study by Ono, Wilkinson, Muter, and Mitson (1972) also found apparent lateral movement regardless of which eye was covered. This was correlated with measured phorias and with the position of the ego-center determined independently by having the subject line up four pairs of lights so that the imaginary straight line between the lights in 
each pair pointed straight at himself. The amount of movement was reasonably well predicted by assuming projection from the independently determined egocenter (located somewhere between the eyes) and taking measured phorias into account. This theory implies an effect of relative dominance which is manifested in the location of the ego-center off the midline, but this effect would be even smaller than that predicted by the Walls-Ogle hypothesis, which places the ego-center in the dominant eye. The failure of the Walls-Ogle hypothesis is somewhat paradoxical in view of the fact that in the simple pointing test of eye dominance, the subject aligns his finger between the target and one eye. One resolution of the paradox is that the instruction to point at a target is not equivalent to the instruction to align one's finger between the target and one's ego-center. If this is correct, then one might expect an effect of dominant eye in visually directed pointing even when the ego-center is located half-way between the eyes.

As is evident in Figure 2, the angular error, if measured from midpoint between the eyes, is largely independent of distance. Angular error is also independent of cue condition and, therefore, of distance error. It shows a small dependence on target distance and a large dependence on the hand used. These hand effects are not consistent across subjects. For four subjects the hands cross, and for the other four they do not. There is some indication that the angular error tends to be toward the side of the favored hand. There is no evidence that it is related to the dominant eye or any other visual characteristic of the subject. It is far too large to be accounted for by phoria.

The existence of a hand effect suggests that angular error is at least in part due to factors other than error in the direction signal produced by the visual system. The same kinds of theoretical interpretations are possible for these indigenous errors as for those produced by prism adaptation (Hardt, Held, \& Steinbach, 1971; Harris, 1965; McLaughlin \& Webster, 1967), and at least some of the same kinds of tests are appropriate. Two subjects in the present experiment participated in one such test. One had shown a leftward error and the other a rightward error. Each pointed "straight ahead" in the absence of any target and also pointed at a single light in the median plane. Each task was done with both the left and the right hands and was repeated six times. In both cases, the error in pointing straight ahead was in the same direction (left or right) as the error in pointing at the target. This confirms the involvement of a nonvisual factor in indigenous angular error.

Apart from the involvement of a nonvisual factor or factors, little can be said about the source of the angular error. These errors, which persisted at least for the 6 days of the experiment, are puzzling in light of the ease with which adaptation to optically induced error may be produced. Why are errors in reaching not substantially eliminated by the same adaptive processes occurring in everyday experience (Held, 1968)? The results of Foley and Held (1972) left open the possibility that the angular error, like the distance error, was diminished in the multicue condition. Consistent with this possibility, they proposed that the adapted state might be situationally specific. However, the finding here that the angular error is independent of cue condition speaks against this hypothesis. Either everyday experience has no effect on pointing errors or this effect is so specific that it does not generalize even to the multicue condition. Either possibility provides a major problem for any theory of perceptual-motor adaptation. Apart from the question of the modifiability of these errors is the equally puzzling question of their origin.

Indigenous angular errors may provide the source of a large amount of variance in prism adaptation studies, even though adaptation is defined as the difference between pre- and postexposure measurements. This effect would be even greater if the exposure conditions correct not only the prisminduced error, but also the indigenous error. This is illustrated by the following example. Suppose a subject who has a leftward error wears a prism which displaces the target by an equal amount to the right. His initial pointing would be accurate and he would not show any adaptation. On the other hand, if the indigenous error is in the same direction as the displacement and both are completely corrected by the exposure condition, then the adaptation effect would exceed the displacement. These possibilities can be tested simply by measuring pointing error before and after adaptation. They suggest the desirability of examining the magnitude of the initial error in prism adaptation studies and perhaps attempting to eliminate it, at least temporarily, prior to adaptation. It seems likely that, for a while after pointing with feedback, a subject will be able to point accurately. Eventually, he may again make consistent errors. Whether a subject returns to the same error pattern after such training is unknown.

\section{REFERENCES}

Bauer, J. A., Woods, G. D., \& Held, R. A device for rapid recording of positioning responses in two dimensions. Behavior Research Methods \& Instrumentation, 1969, 1, 157-159.

Foley, J. M., \& HeLd, R. Visually directed pointing as a function of target distance, direction and available cues. Perception \& Psychophysics, 1972, 12, 263-268.

Gogel, W. C. Scalar perceptions with binocular cues of distance. American Journal of Psychology, 1972, 85, 477.497. 
Hardt, M. E., Held, R., \& Steinbach, M. J. Adaptation to displaced vision: A change in the central control of sensorimotor coordination. Journal of Experimental Psychology, $1971,89,229-239$.

Harris, C. Perceptual adaptation to inverted, reversed, and displaced vision. Psychological Review, 1965, 72, 419-444.

HELD, R. Action contingent development of vision in neonatal animals. In D. F. Kimble (Ed.), Experience and capacity. New York: New York Academy of Sciences, 1968.

McLaughlin, S. C., \& Webster, R. G. Changes in straightahead eye position during adaptation to wedge prisms. Perception \& Psychophysics, 1967, 2, 37-44.
OGLE, K. N. The optical space sense. In H. Davson (Ed.), The eye (Vol. 4). New York: Academic Press, 1962. Pp. 209-417. Ono, H., Wilkinson, A., Muter, P., \& Mitson, L. Apparent movement and change in perceived location of a stimulus produced by a change in accommodative vergence. Perception \& Psychophysics, 1972, 12, 187-192.

Walls, G. L. A theory of ocular dominance. Archives of Ophthalmology, 1951, 45, 387-412.

(Received for publication April 17, 1974; revision received August 12, 1974.) 